\title{
EFFECT OF LEE-WICK THERMODYNAMICS IN THE COSMOLOGY OF THE EARLY UNIVERSE
}

\author{
KAUSHIK BHATTACHARYA \\ Department of Physics, Indian Institute of Technology, Kanpur, \\ Kanpur 208016, India, \\ kaushikb@iitk.ac.in \\ SURATNA DAS \\ Tata Institute of Fundamental Research, Homi Bhabha Road, \\ Colaba, Mumbai 400005, India \\ suratna@tifr.res.in
}

\begin{abstract}
The Lee-Wick theories require unusual Lee-Wick (LW) partners to the standard model (SM) particles. The excitations of the unusual fields may have indefinite norms in the Hilbert space. In the present talk the thermodynamic results of a toy LW (where each normal particle has only one LW partner and the standard massless gauge fields do not appear in the normal particle spectrum as their LW partners has the potential to produce negative energy density) as well as a realistic LW universe (where the above restrictions are not present) will be discussed.
\end{abstract}

\section{The toy model}

To tackle the problem of divergences in quantum field theories (QFTs) the LW theories 1 assume a LW partner for each SM field. It turns out that to attack the problem of divergences in QFT one has to quantize the LW partners in such a way that their excitations may have indefinite norms in the Hilbert space. In the present talk we will utilize the LW standard mode ${ }^{2}$ to investigate the physics of the early universe and we will mainly follow Ref. 3 .

In the toy model it is assumed that each SM field is accompanied by a LW partner. In this simplified picture the energy density, pressure for each of these partners can be calculated ${ }^{\sqrt[45]{5}}$ As the LW partner states can have indefinite norm, they are barred to appear as in-states or out-states in any S-matrix process. The LW partners can only appear as resonances in processes involving SM particles. The LW resonances may thermalize (in the cosmic plasma) if the SM particles energies are high i.e., $T>M$. If $T<M$ the LW resonances decouple and turn out to be non-thermal. If $\tilde{M}$ is the mass of the maximally massive partner field whose generic mass is $M$ which is less than $T$, then the net energy density and entropy density of the relativistic fields in the early universe composed of the SM fields and there LW partners turns out to be,

$$
\rho=\frac{\tilde{M}^{2}}{24} \tilde{g}_{*} T^{2}, \quad s=\frac{\tilde{M}^{2}}{12} \tilde{g}_{* s} T
$$

where $\tilde{g}_{*}$ is the effective degree of freedom for energy calculation, and $\tilde{g}_{* s}$ is the effective degree of freedom for entropy calculation. Here it has been assumed that 
each SM boson (or fermion) and its LW partner are in thermal equilibrium but all the different species of particles and their partners may not be sharing the same temperature. In the above relations $\tilde{M}$ is the mass of the maximally massive $\mathrm{LW}$ partner which is relativistic at temperature $T$. The above equations for energy density and entropy density are correct if the spectrum of the particles does not contain any massless gauge bosons. In presence of massless gauge bosons, in the realistic model, the above relations get modified.

Using the above forms of energy density and entropy density one can show the time-temperature relation for the LW universe to be:

$$
T_{\mathrm{GeV}} \sim 10^{-24}\left(\pi \tilde{g}_{*}\right)^{-1 / 2}\left(\frac{M_{\mathrm{Pl}}}{\tilde{M}}\right) \frac{1}{t_{\mathrm{sec}}}
$$

The time-temperature relation is plotted in Fig. 1. The bump appears at a specific time in Fig. 11 because at that time the heaviest LW partner with mass $\tilde{M}$ becomes non-relativistic and the the heaviest mass value in Eq. (2) is replaced by the next heaviest LW partner mass $\tilde{M}^{\prime}$. This is an out of equilibrium process as the entropy also shoots up during this time. As the LW partners become non-relativistic they decouple from the plasma and do not contribute in the energy density or entropy density of the system.

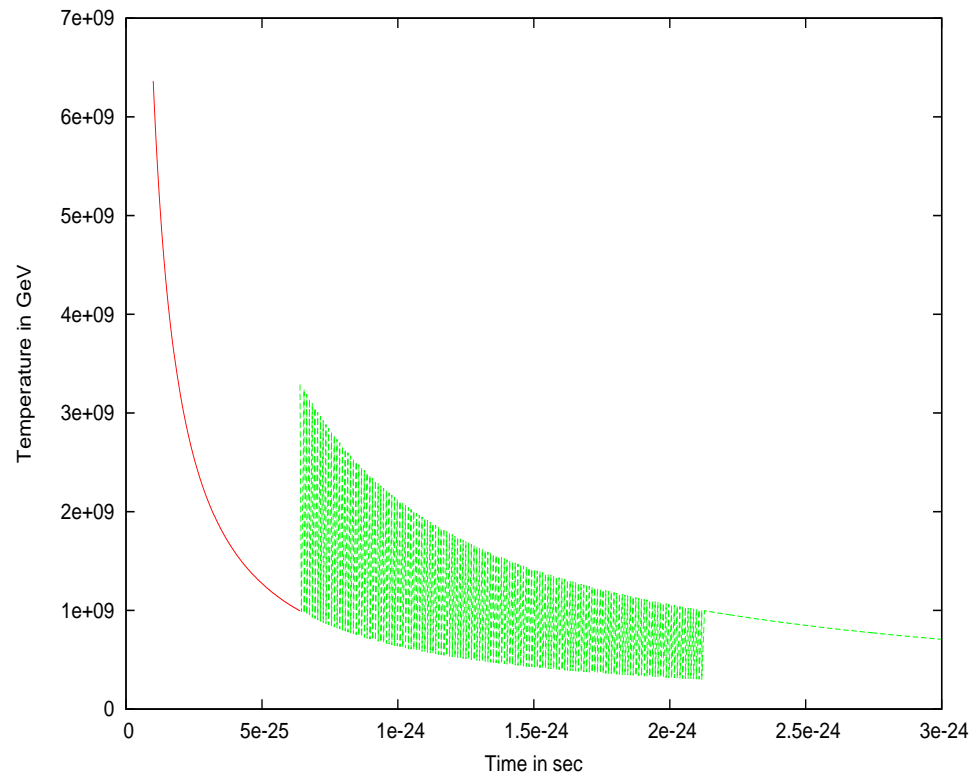

Fig. 1. Figure showing the bump in the temperature as the heaviest Lee-Wick partner with mass $10^{9} \mathrm{GeV}$ becomes non-thermal and its position is taken up by the next heaviest Lee-Wick partner of mass $3 \times 10^{8} \mathrm{GeV}$. For a simple illustration the effective degrees of freedom are assumed to remain constant in the process, $\tilde{g}_{*} \sim 80$. The green region in the plot represents a region of rapid and violent temperature fluctuations where the system has gone out of equilibrium. 


\section{The realistic model}

In the realistic model there are two fermionic partners of a standard chiral fermion and there is one more degree of freedom for the massive partners of the massless gauge bosons. In this case:

$$
\rho=\frac{\tilde{M}^{2}}{24} \tilde{g}_{* N} T^{2}-\frac{7 \pi^{2}}{240} \tilde{g}_{F} T^{4}-\frac{\pi^{2}}{30} n T^{4},
$$

where the new degrees of freedom $\tilde{g}_{* N}$ originates from the standard bosonic contribution. As there are more LW fermions, the unpaired fermionic contribution comes with $\tilde{g}_{F}$. The last term in Eq. (3) arises from the extra longitudinal degree of freedom of the massive LW partners of the normal massless gauge bosons. Here $n$ denotes the number of massive vector boson partners of SM gauge bosons. In the last equation it is assumed that all the species in the plasma are in thermal equilibrium.

In the present scenario one sees that if there are two LW fermionic partners for each standard fermion and the longitudinal mode of the LW partners of the normal gauge bosons also contribute to the energy density then the net energy density is not positive definite. There can be two ways in which one can obtain a positive definite energy density of the early universe infested with LW partners. The first one depends upon the principle of parametric resonance during preheating after inflation. ${ }^{6}$ During these phase only massive bosons are produced and if these massive bosons initially thermalize during reheating then the problem with the fermions and gauge bosons disappear. The second option is related to the reheating temperature of the universe. If the LW fermion partners and the LW partners of the gauge bosons decoupling temperature is more than the reheat temperature then both of these species will not be present in the energy density term and henceforth the energy density will be positive definite. These scenario depends upon the mass hierarchy of the LW partners.

In conclusion one can say that a realistic LW radiation dominated universe is a difficult thing to realize but for certain circumstances as discussed above one can indeed have a LW radiation phase during/after reheating.

\section{References}

1. T. D. Lee and G. C. Wick, Phys. Rev. D 2, 1033 (1970).

2. B. Grinstein, D. O'Connell and M. B. Wise, Phys. Rev. D 77, 025012 (2008) arXiv:0704.1845 [hep-ph]].

3. K. Bhattacharya and S. Das, Phys. Rev. D 86, 025009 (2012) arXiv:1203.1109 [hep$\mathrm{ph}]]$.

4. B. Fornal, B. Grinstein and M. B. Wise, Phys. Lett. B 674, 330 (2009) arXiv:0902.1585 [hep-th]].

5. K. Bhattacharya and S. Das, Phys. Rev. D 84, 045023 (2011) arXiv:1108.0483 [hep$\mathrm{ph}]$.

6. L. Kofman, A. D. Linde and A. A. Starobinsky, Phys. Rev. Lett. 73, 3195 (1994) hep-th/9405187. 